This is a postprint version of the following published document:

Castro, R. Gago, L. Vázquez, J. Muñoz-García, and R. Cuerno, "Stressinduced solid flow drives surface nanopatterning of silicon by ion-beam irradiation". In Physical Review B 86 (2012) 19 December, 214107. Available in http://dx/doi.org/10.1103/PhysRevB.86.214107

(c) 2012 American Physical Society 


\title{
Stress-induced solid flow drives surface nanopatterning of silicon by ion-beam irradiation
}

\author{
M. Castro, ${ }^{1, *}$ R. Gago, ${ }^{2}$ L. Vázquez, ${ }^{2}$ J. Muñoz-García, ${ }^{3}$ and R. Cuerno ${ }^{3}$ \\ ${ }^{1}$ Grupo Interdisciplinar de Sistemas Complejos (GISC) and Grupo de Dinámica No Lineal (DNL), Escuela Técnica Superior de Ingeniería \\ (ICAI), Universidad Pontificia Comillas, E-28015 Madrid, Spain \\ ${ }^{2}$ Instituto de Ciencia de Materiales de Madrid, Consejo Superior de Investigaciones Científicas, E-28049 Madrid, Spain \\ ${ }^{3}$ Departamento de Matemáticas and Grupo Interdisciplinar de Sistemas Complejos (GISC), Universidad Carlos III de Madrid, \\ Avenida de la Universidad 30, E-28911 Leganés, Spain \\ (Received 27 February 2012; revised manuscript received 8 November 2012; published 19 December 2012)
}

\begin{abstract}
Ion-beam sputtering (IBS) is known to produce surface nanopatterns over macroscopic areas on a wide range of materials. However, in spite of the technological potential of this route to nanostructuring, the physical process by which these surfaces self-organize remains poorly understood. We have performed detailed experiments of IBS on Si substrates that validate dynamical and morphological predictions from a hydrodynamic description of the phenomenon. We introduce a systematic approach to perform the experiments under conditions that guarantee the applicability of a linear description, helping to clarify the experimental framework in which theories should be tested. Among our results, the pattern wavelength is experimentally seen to depend almost linearly on ion energy, in agreement with existing results for other targets that are amorphous or become so under irradiation. Our work substantiates flow of a nanoscopically thin and highly viscous surface layer, driven by the stress created by the ion beam, as an accurate description of this class of systems.
\end{abstract}

PACS number(s): 81.16.Rf, 05.45.-a, 68.35.Ct, 79.20.Rf

\section{INTRODUCTION}

In the paper that historically coined the word "nanotechnology,"1 erosion of solid targets through ion-beam sputtering (IBS) was already put forward as the most promising technique to structure the surface of a wide range of materials. Indeed, for ion energies ranging from $100 \mathrm{eV}$ to $100 \mathrm{keV}$, IBS has shown a remarkable capability ${ }^{2}$ to produce ordered nanoscale-sized patterns (mostly ripples and dots) over large areas (up to tens of $\mathrm{cm}^{2}$ ) for a wide range of targets, including semiconductors, metals, and insulators. ${ }^{3,4}$ However, despite its large potential for technological applications, ${ }^{5}$ the promise of IBS as a fully controlled and understood method to tailor patterns with custom-designed properties has turned out hard to achieve. This is partly due to the huge time-scale separation among different processes that influence the system, which complicates the clear-cut identification of the main underlying physical mechanisms.

Classically, since the seminal work by Bradley and Harper ${ }^{6}$ (BH) the interplay between sputtering and surface diffusion had been identified as the key mechanism leading to pattern formation in IBS. Thus, a characteristic length scale would be selected $^{7}$ from the competition between the morphologically unstable dependence of the sputtering yield with local surface curvature and thermal surface diffusion that smooths out surface features. However, thus far only partial qualitative agreement has been reached between this classical description and experiments. ${ }^{3}$ After the recent realization of the nontrivial role of impurities in the emergence of the pattern for the simplest case of monoelemental semiconductor targets like silicon, ${ }^{8,9}$ it has been necessary to go beyond the $\mathrm{BH}$ mechanism, as shown in recent experiments on $\mathrm{Ar}^{+}$irradiation. ${ }^{10,11}$ For instance, one of the most direct implications of the $\mathrm{BH}$ picture, that pattern formation should take place for any incidence angle $\theta$ between the ion beam and the normal to the target, has been experimentally disproved, there being a critical angle $\theta_{c}$ such that the (ripples) pattern appears only for $\theta>\theta_{c}$. Working at ion energies between 10 and $40 \mathrm{keV}$, higher than in Refs. 10 and 11, this fact was already noted by Carter and Vishnyakov ${ }^{12}(\mathrm{CV})$, who explained it phenomenologically via a smoothing effect of momentum transfer from the ions to the target atoms.

This new scenario poses the need to perform systematic and controlled experiments on a model system such as clean silicon, and quantitatively contrast the experimental data with theoretical predictions. To date, two main non-BH-type mechanisms have been proposed to explain formation or absence of patterns in IBS of monoelemental semiconductors, namely, mass redistribution ${ }^{13-16}$ and ion-induced solid flow. ${ }^{17-19}$ The former employs results from molecular dynamics (MD) simulations in order to rephrase the Carter-Vishnyakov effect ${ }^{12}$ as the influence on the surface morphology of mass (rather than momentum) transfer induced by the beam. The solid flow model proceeds, rather, through a continuum description of the surface flow that is driven by the confined stress due to the accumulated damage on the surface amorphous layer produced by irradiation. Available models for flow include both Newtonian ${ }^{17-19}$ and viscoelastic ${ }^{20,21}$ constitutive laws.

Remarkably both the mass redistribution and the viscous flow descriptions agree with experiments with respect to, e.g., the value of $\theta_{c}$ and the dependence of the pattern wavelength on $\theta \cdot{ }^{18}$ Note that, as far as physical mechanisms are concerned, MD simulations ${ }^{13-16}$ are fundamentally limited in the IBS context, since they cannot probe the required macroscopic time scales at which nontrivial surface evolution takes place. This limitation has required, e.g., the ad hoc use of surface confined viscous flow ${ }^{22}$ in Refs. 14 and 15 , to obtain the correct time scales for the description of the dynamics.

In this paper, we describe IBS experiments on $\mathrm{Si}$ in order to validate specific predictions, from the hydrodynamic description put forward in Ref. 19, that stem from the assumption that the main driving field in the process is the ion-induced residual stress that builds up in the flowing layer. Particular 
care has been taken to perform the experiments under wellcontrolled conditions that allow their meaningful comparison with the theoretical predictions. Among our results, we obtain that the ripple wavelength $\lambda$ depends almost linearly on the ion energy $E$. This relation for $\mathrm{Si}$ is akin to similar $\lambda$ vs $E$ behaviors reported for silica, ${ }^{22}$ highly oriented pyrolitic graphite (HOPG), ${ }^{23}$ or amorphous carbon, ${ }^{24}$ all of which are amorphous or become amorphized by the irradiation. ${ }^{25}$ Consistency between experiments and theoretical predictions thus allows us to elucidate the physical mechanism through which pattern formation occurs in this class of nonequilibrium nanoscopic systems.

\section{PREDICTIONS OF THE THEORY}

The solid flow description ${ }^{19}$ of IBS is based on the fact that, as a consequence of the impact of the ions and the subsequent release of energy within the target, defects are created inside the material. These events occur in a few picoseconds after the impact. Partial relaxation of the defects leads to sputtering of target atoms, but also to the generation of a residual stress that is confined to a thin amorphous layer that builds up beneath the surface $\mathrm{e}^{26-28}$ and that reaches a stationary thickness after the very early times of irradiation. This ion-induced (compressive) stress is characterized by a slow time relaxation that involves highly viscous flow of the amorphous layer, which will be assumed to be incompressible (see Fig. 1 for a schematic

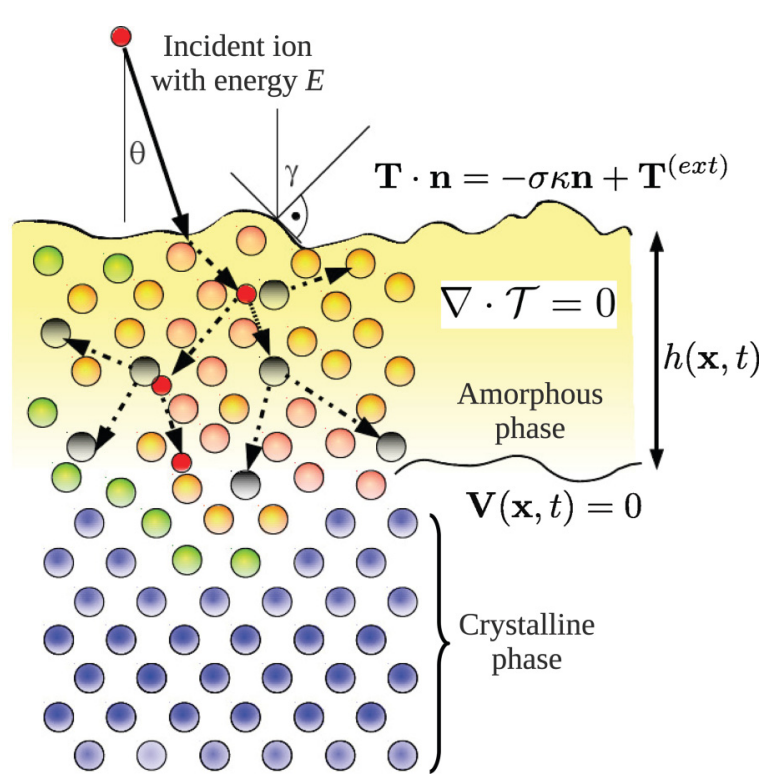

FIG. 1. (Color online) Schematic view of the IBS process. An incident energetic ion (small red circle) impinges onto the target at an angle $\theta$, inducing a collision cascade that amorphizes a thin region through creation of vacancies and interstitials. At long time scales, the amorphized solid flows as a highly viscous fluid. Here $\gamma$ is the local slope of the surface profile $h(\mathbf{x}, t)$ at time $t$ and point $\mathbf{x}, \mathcal{T}=\mathbf{T}-\mathbf{T}^{(\mathrm{ext})}$ with $\mathbf{T}$ the fluid stress tensor and $\mathbf{T}^{(\mathrm{ext})}$ the stress induced by the irradiation process, $\mathbf{V}$ is the fluid velocity, and $\sigma \kappa$ is the contribution from surface tension. Different colors are meant to represent the degree of order and kinetic energy. representation of the IBS process, and Ref. 19 for notation and a detailed explanation of the essentials of the theory).

The real part of the linear dispersion relation [namely, the rate at which periodic perturbations with wave vector $q$ of a flat profile, $h(x)=$ const, grow or decay $\left.{ }^{7}\right]$ is given by ${ }^{19}$

$$
\omega_{q}^{\prime}=-\frac{f_{E} d^{3} \phi(\theta)}{3 \mu} q^{2}-\frac{\sigma d^{3}}{3 \mu} q^{4},
$$

where $\phi(\theta)=\partial_{\theta}[\Psi(\theta) \sin (\theta)], d$ is the average thickness of the amorphous layer, $\sigma$ is the surface tension (energy), and $\mu$ is the (ion-induced) viscosity. Note that, in our range of interest for ion energies (say, 300 to $1000 \mathrm{eV}$ ), the ripple wavelength (tens of nanometers) is much larger than the thickness of the amorphous layer (a few nanometers). . $^{3,4}$

In Eq. (1), the parameter $f_{E}$ can be understood as the gradient of residual stress induced by the ions across the amorphous layer, whose angular dependence is described through the function $\Psi(\theta)$. In order to test the theory and provide further predictions, the function $\Psi(\theta)$ needs to be determined in a form that is compatible with experiments or MD simulations. Thus, as the sign of the incidence angle does not change the wavelength of the pattern, $\Psi$ has to be an even function of $\theta$. In addition, as at grazing incidence the ion does not interact with the surface, $\Psi(\theta \rightarrow \pi / 2) \rightarrow 0$. In summary, $\Psi$ can be written as a cosine Fourier series of $\theta$. Here, we take $\Psi(\theta)=\cos \theta$ (namely, we just consider the first term in the Fourier series) as the simplest choice that leads to the expected local reduction of the ion-beam flux by a factor $\cos (\theta-\gamma)$ as a function of the surface slope, $\gamma=\arctan \partial_{x} h .^{12,19}$ Such a choice leads to good agreement ${ }^{19}$ with previous experiments, as well as with the ones presented below.

Given $\Psi$ and $f_{E}$, testable predictions can be made through the value of the ripple wavelength $\lambda,{ }^{19}$

$$
\lambda=2 \pi \sqrt{\frac{2 \sigma}{-f_{E} \phi(\theta)}} .
$$

The first prediction has to do with the angular dependence of the patterns. For $\Psi(\theta)=\cos \theta$ [hence, $\phi(\theta)=\cos (2 \theta)]$, we find a value for the critical angle $\theta_{c}=45^{\circ}$. This critical angle at $45^{\circ}$ can also be obtained ${ }^{19}$ without assuming a specific functional form for $\Psi(\theta)$ and assuming that, instead of a body force created by the ion, there is an elastic stress confined at the free surface for an incompressible material. ${ }^{19}$ In addition to the value itself, it is remarkable that this angle does not depend on the ion energy, in good agreement with x-ray experiments, ${ }^{14}$ and in contrast with the energy-dependent critical angle that is obtained from MD simulations in Ref. 15, from $\theta_{c} \simeq 40^{\circ}$ at $250 \mathrm{eV}$ to $\theta_{c} \simeq 35^{\circ}$ at $100 \mathrm{eV}$.

Up to this point, the kinetics of the pattern formation process is correctly captured within the viscous flow framework. However, we have not yet detailed the process that is causing flow to occur in the first place, which would constitute the underlying physical mechanism ultimately inducing the pattern formation. In principle, we attribute it to the stress that sets in throughout the amorphous layer as a consequence of the damage that is induced by the collision cascades occurring in this range of energies. Viscous flow is then the response of the system, trying to relax the external driving thus exerted by irradiation. Our aim is to enquire into the further implications 
of our assumption on the nature of the driving, in order to assess it on a solid basis.

A simple model based on linear collision cascades is available, ${ }^{29}$ in which the stress that is induced by irradiation damage follows, through Hooke's law, from the volumetric strain produced by atom displacements from their equilibrium positions. The magnitude of this stress actually depends on the ion average energy, following a square-root law ${ }^{29} T^{(\mathrm{ext})} \sim$ $E^{1 / 2}$, which becomes modified if one takes into account the partial stress relaxation that occurs due to defect migration. ${ }^{30}$ Such a relaxation takes place in a time scale that is comparable to the one needed for collision cascades to complete, which in turn is orders of magnitude faster than the macroscopic time scales in which viscous flow takes place. Specifically, Davis $^{30}$ assumed spike formation to allow displaced atoms to move to the free surface and thus decrease the effective stress generation, leading to

$$
T^{(\mathrm{ext})}(E) \propto \frac{Y}{1-v} \frac{E^{1 / 2}}{R / J+\tilde{\rho} E^{5 / 3}} .
$$

Here, $Y$ and $v$ are the material Young's and Poisson's moduli, and $R$ and $\tilde{\rho}$ are other material-dependent constants (related to properties like the binding energy) so that, in the energy range we are considering, ${ }^{30} T^{\text {(ext) }}(E) \sim E^{-7 / 6}$.

Although spike formation has been shown to account for experiments done at energies down to the $50 \mathrm{eV}-1 \mathrm{keV}$ range, ${ }^{31}$ it is usually believed that processes at these energies are better described by a binary-collision picture. ${ }^{25}$ Nevertheless, as shown in Ref. 32, detailed binary-collision simulations in which electronic stopping effects are also taken into account lead to an energy dependence of the generated stress that cannot be distinguished from that in Ref. 30 for energies below $2 \mathrm{keV}$. Hence, we employ the latter for analytical convenience leading, for the energy range in our experiments, to $f_{E} \propto E^{-7 / 6-2 m}$, where we have assumed that the thickness of the amorphous layer also scales with energy, as $d \sim E^{2 m}$, the value of $m$ usually being between $1 / 3$ and $1 / 2$ as determined from TRIM simulations. ${ }^{33}$ Taking into account the spread in the latter exponent value, our assumption on stress as the driving force for the evolution of the target surface leads, through Eq. (2), to a dependence of the ripple wavelength on energy as

$$
\lambda \simeq E^{0.92}-E^{1.08} \approx E .
$$

In a similar way to the critical angle, this proportionality of $\lambda$ with $E$ can be also obtained assuming that, instead of a body force created by the ion, there is an elastic stress confined at the surface for an incompressible material. ${ }^{19}$ Note that a close-to linear relation between ripple wavelength and ion energy contradicts predictions based on the $\mathrm{BH}$ mechanism. ${ }^{3,4}$ For $\mathrm{Ar}^{+}$irradiation at the ion energies we are considering here, such a linear dependence of the wavelength on ion energy has already been reported for surfaces other than $\mathrm{Si}$, such as $\mathrm{SiO}_{2}$ (Ref. 22) - for which surface-confined viscous flow was explicitly advocated in order to account for the observed $\lambda(E)$ law-, graphite, ${ }^{23}$ and amorphous carbon. ${ }^{24}$ In the case of Si, previous assessments of the $\lambda$ vs $E$ relation available in the literature seem possibly affected by contamination issues.

A further prediction can be obtained from Eq. (1), that allows one to find the characteristic time scale $\tau$ for the exponential growth of the pattern amplitude occurring at short times where the linear approximation holds. Indeed, under conditions for pattern formation and within the linear approximation, there is a single Fourier mode $q^{*}$ whose amplitude dominates all other modes exponentially in time, $h_{q^{*}}(t) \propto e^{\omega_{q^{*}}^{\prime} t}$, leading to the formation of a ripple structure with wavelength $\lambda=2 \pi / q^{*}$. Thus,

$$
\tau(E, \theta) \equiv 2 \pi / \omega_{q^{*}}^{\prime} \sim \frac{\mu}{f_{E}^{2} d^{3} \phi^{2}(\theta)} \sim \frac{E^{b}}{J \phi^{2}(\theta)}
$$

is the smallest time scale associated with linear ripple formation, being given by the rate of growth of the fastest developing perturbation. In Eq. (5) we have used the same scaling law for $f_{E}$ and $d$ as above to get $b \in[1.33,1.67]$ and that $\mu \sim 1 / J .^{22}$ Notice that, e.g., the surface roughness $W$ grows in the linear unstable regime as $W \sim h(t) \propto e^{t / \tau}$. This exponential behavior will eventually be interrupted by nonlinear mechanisms (coming, for instance, from stress ${ }^{19}$ or from purely erosive effects ${ }^{34-36}$ ) at sufficiently long times. Hence, the time duration of the validity of the linear approximation is also safely characterized by $\tau$. In particular, the above formula implies that such duration depends on system parameters, such as average flux, energy, and angle of incidence.

\section{EXPERIMENTS}

Commercial single-crystal $\mathrm{Si}(100)$ targets (380 $\mu \mathrm{m}$ thick, $p$ type B doped, 1-10 $\mathrm{m} \Omega \mathrm{cm}$ ) were sputtered with $\mathrm{Ar}^{+}$ ions under different incidence angles within the 300-1100 eV energy range. The angle $\theta$ was adjusted by axial rotation of the sample in front of the ion gun with an overall resolution of $\pm 3^{\circ}$. The ions were extracted from a commercial 3-cm-beamdiameter Kaufman-type ion gun (VEECO) located $25 \mathrm{~cm}$ away from the target. In order to avoid metal contamination on the surface during irradiation, the sample holder was covered with a sacrificial Si wafer and the sample was attached to it with a double-sided conductive vacuum tape. Prior to the process, the current density at the sample position in the plane parallel to the source grids $\left(J_{0}\right)$ was set to $300 \mu \mathrm{A} \mathrm{cm}{ }^{-2}$ (constantdose experiments; see below) or $30 \mu \mathrm{A} \mathrm{cm}^{-2}$ (intrinsic-time experiments) with a Faraday cup located on a movable shutter that prevents, additionally, unwanted irradiation of the sample. The base pressure of the system is $<10^{-6}$ mbar and the working pressure is $2 \times 10^{-4}$ mbar using highly pure $\operatorname{Ar}(99.99995 \%)$. The lack of heavy impurities in the irradiated samples has been checked using standard x-ray photoemission spectroscopy. It is worth mentioning that light contaminants from the residual vacuum such as $\mathrm{C}$ or $\mathrm{O}$ are present in very low concentrations and, in any case, they do not affect the resulting morphologies. The surface morphology of the irradiated surfaces was imaged ex situ with a Nanoscope IIIa equipment (Bruker) operating in intermittent-contact mode and using silicon cantilevers (Bruker) with a nominal radius of curvature of $8 \mathrm{~nm}$.

In Fig. 2 we show four atomic force microscopy (AFM) micrographs of $\mathrm{Si}$ (100) surfaces bombarded at $E=700 \mathrm{eV}$ at a constant dose of $6 \times 10^{17}$ ions $/ \mathrm{cm}^{2}$ and different incidence angles. The surface does not contain any visible pattern for $\theta<50^{\circ} \pm 3^{\circ}$ (not shown). On the other hand, as seen in Fig. 3(a), roughness values increase significantly above this critical angle. ${ }^{11}$ Figures 2(b) through 2(d) show patterns that are less and less sinusoidal in shape, suggesting an augmented 

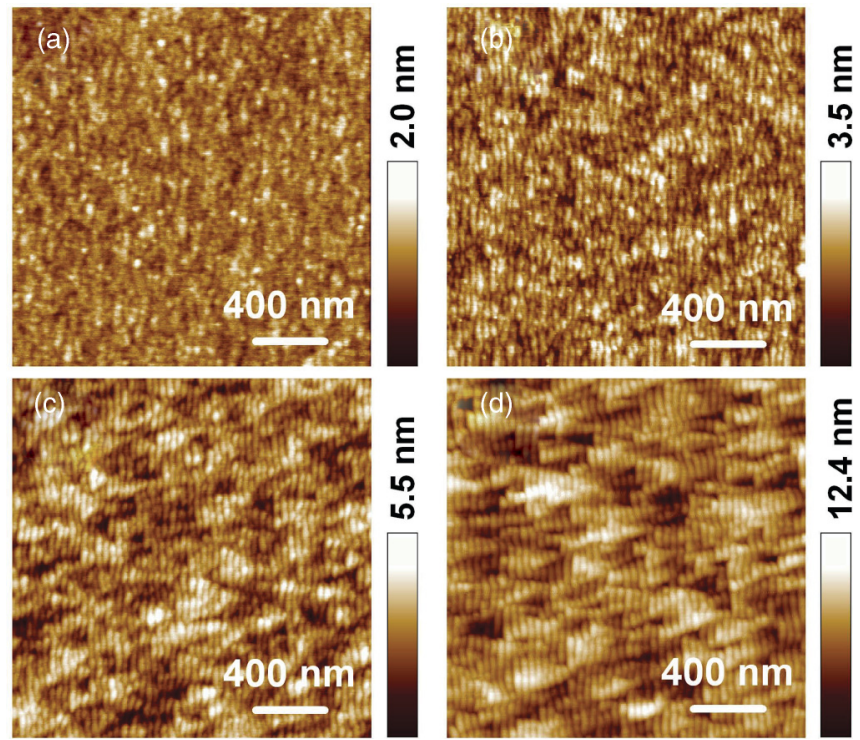

FIG. 2. (Color online) Experimental morphologies of ion irradiation of $\mathrm{Ar}^{+}$on $\mathrm{Si}$. All the figures are obtained at energy $E=$ $700 \mathrm{eV}$, constant dose of $6 \times 10^{17} \mathrm{ions} / \mathrm{cm}^{2}$, and at angles of incidence (a) $\theta=55^{\circ}$, (b) $\theta=60^{\circ}$, (c) $\theta=65^{\circ}$, and (d) $\theta=70^{\circ}$. For $\theta<$ $50^{\circ} \pm 3^{\circ}$, no pattern is observed (not shown). Ions arrive from right to left in all cases.

dominance of nonlinear effects when $\theta$ is increased at a fixed fluence. For $\Psi(\theta)=\cos \theta$, we have $\phi(\theta)=\cos (2 \theta)$ and this observation agrees with what would be expected from Eq. (5), namely, the linear instability develops on a faster time scale for increasing $\theta$, leading to earlier onset of nonlinear effects.

Figure 4 provides a complementary view of the morphology, namely, its explicit time evolution. Thus, topographies are shown for fixed $\theta=65^{\circ}$ and $E=500 \mathrm{eV}$, and for increasing fluences (a) to (d). We see that, e.g., the long-time morphology in Fig. 4(d) that features faceted shapes ${ }^{37}$ looks qualitatively similar to Fig. 2(d), suggesting that the latter corresponds to a dynamical regime that is more long time than that of Fig. 2(b), which is in turn similar to Fig. 4(b).

We can actually make a more quantitative comparison between experimental data and model predictions as derived above, through Eq. (5). As expressed by this formula and as has just been qualitatively discussed in view of the experimental morphologies, the linear regime is expected to have a duration that changes with experimental conditions. Thus, it would be shorter for increasing angles of incidence $\theta>\theta_{c}$, in a form reminiscent of critical slowing down for continuous phase transitions; ${ }^{7,18}$ note that the morphological transition at $\theta=\theta_{c}$ is precisely of this type. ${ }^{10}$ Actually, Eq. (5) allows us to control the experimental times, in order to guarantee that the system is truly evolving within the same stage of the linear regime, given a fixed reference experiment in which such a state can be unambiguously assessed. The use of this intrinsic time scale allows a similarity relation to be established between different experiments. ${ }^{38}$ Thus, given a pair of angle-energy reference values, $\left(\theta_{\text {ref }}, E_{\text {ref }}\right)$, we can extrapolate the value of $\tau$ for any other pair $(\theta, E)$ through

$$
\tau(\theta, E)=\tau\left(\theta_{\mathrm{ref}}, E_{\mathrm{ref}}\right) \frac{J_{\mathrm{expt}}\left(\theta_{\mathrm{ref}}, E_{\mathrm{ref}}\right) E_{\mathrm{ref}}^{-7 / 3+2 m} \phi^{2}\left(\theta_{\mathrm{ref}}\right)}{J_{\mathrm{expt}}(\theta, E) E^{-7 / 3+2 m} \phi^{2}(\theta)},
$$
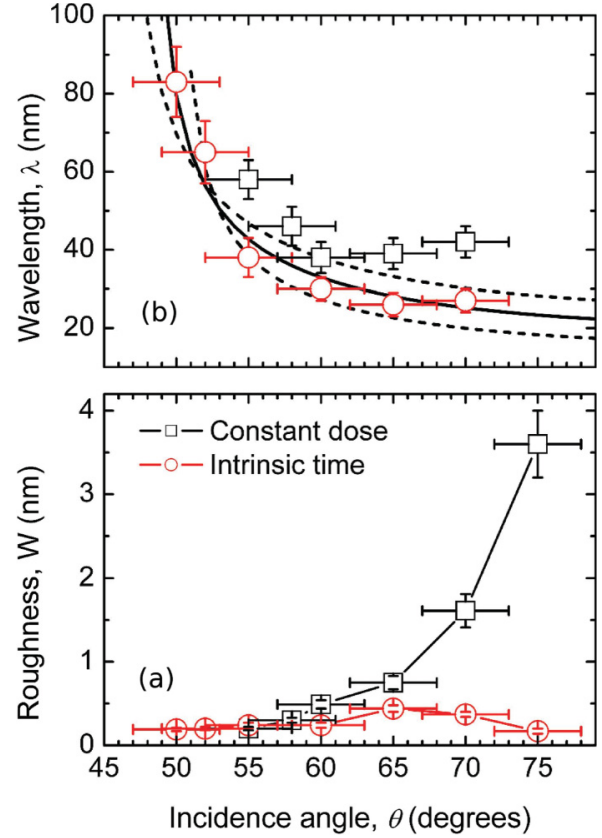

FIG. 3. (Color online) (a) Roughness and (b) wavelength dependence on incidence angle for $E=700 \mathrm{eV}$. Squares were obtained at a constant dose of $6 \times 10^{17}$ ions $/ \mathrm{cm}^{2}\left(J_{0}=300 \mu \mathrm{A} \mathrm{cm}{ }^{-2}\right)$ and circles correspond to experiments performed in the proper linear regime $\left(J_{0}=30 \mu \mathrm{A} \mathrm{cm}^{-2}\right)$ as estimated by the intrinsic time scale in Eq. (5). Lines in (a) are guides to the eye. The solid line in (b) is a fit to Eq. (2), the dashed lines corresponding to the same fit but assuming a $\pm 3^{\circ}$ uncertainty in $\theta$.

where $J_{\text {expt }}(\theta, E)=J_{0}(E) \cos \theta$ is the flux used in a particular experiment at energy $E$ and angle $\theta$. In our case, we choose $\theta_{\text {ref }} \equiv 65^{\circ}$ and $E_{\text {ref }} \equiv 700 \mathrm{eV}$. Thus, from Eq. (6) we can extract the experimental times for different angles or energies which we define as the intrinsic time scale, namely, the time at which experiments at different angles and/or energies are within the linear regime.

In Fig. 3(a) we show how the surface roughness depends on the conditions under which the experiments have been performed. Thus, we emphasize that doing the experiments at constant time (or dose or fluence) may produce surfaces which are described by different regimes (linear or nonlinear), depending on the value of $\theta$. The morphologies in Fig. 2 correspond to the times obtained at constant dose in Fig. 3 where, as mentioned, typical nonlinear motifs, like facets, are recognized, specially in Figs. 2(c) and 2(d). Note how the onset of these nonlinear effects correlates for different observables, ${ }^{4}$ like the power-law growth of $W$ or the coarsening of $\lambda$ in Fig. 5, both of which are beyond linear approximation. Indeed, notice on the one hand that the amplitudes of the height Fourier modes, and thus the roughness, grow or decay exponentially in time within the linear regime. On the other hand, the coefficients in the linear dispersion relation are time independent, so that coarsening cannot be obtained within such a regime.

In addition, in Fig. 3(b) we show the dependence of the ripple wavelength on the incidence angle. The squares stand for experiments performed at constant dose and the circles are for experiments at the intrinsic time scale defined in Eq. (5) that 

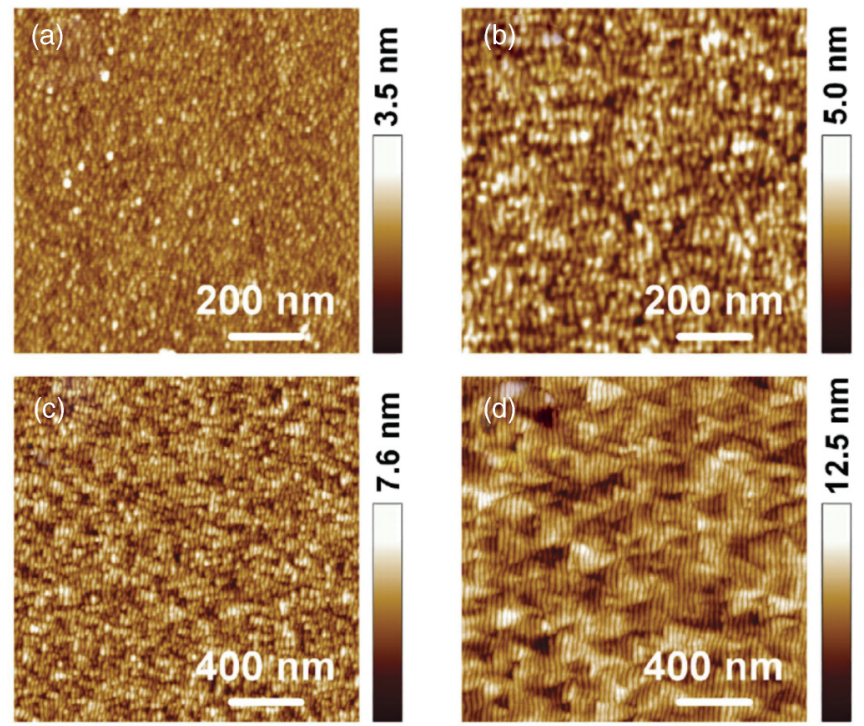

FIG. 4. (Color online) Experimental dynamics for ion irradiation of $\mathrm{Ar}^{+}$on $\mathrm{Si}$. The figures are obtained at fixed energy $E=500 \mathrm{eV}$, flux $J=30 \mu \mathrm{A} \mathrm{cm}^{-2}$, and constant angle of incidence $\theta=65^{\circ}$, at increasing irradiation times $t=$ (a) $3 \mathrm{~min}$, (b) $15 \mathrm{~min}$, (c) $30 \mathrm{~min}$, and (d) $120 \mathrm{~min}$. Ions arriving from right to left in all cases.

guarantees linear behavior. The solid line corresponds to a fit to Eq. (2) using $\Psi(\theta)=\cos \theta$, and the dashed lines are a similar fit taking into account a $\pm 3^{\circ}$ uncertainty in the experimental measurement of $\theta .{ }^{39}$ These fits confirm the validity of Eq. (2).
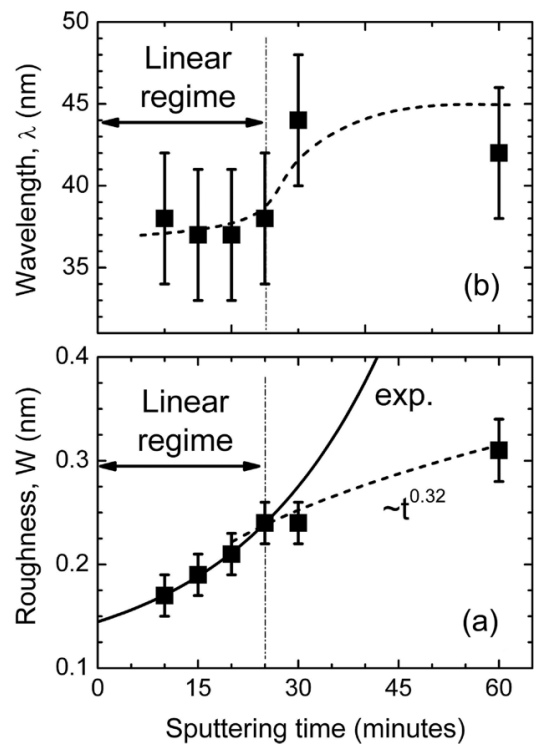

FIG. 5. Time evolution of the (a) surface roughness and (b) ripple wavelength for $E=700 \mathrm{eV}, J_{0}=30 \mu \mathrm{A} \mathrm{cm}^{-2}$ and $\theta=55^{\circ}$. The solid line in (a) is an exponential fit in the linear regime, while the dashed line is a power-law fit in the nonlinear regime with exponent 0.32 . The dashed line in (b) is a guide to the eye. The time regime in which the wavelength grows with time (coarsening) starts at $t \simeq$ $25 \mathrm{~min}$, as indicated by the vertical dotted line; this is a signature of the onset of nonlinear effects. At shorter times the wavelength is almost constant. The same crossover time separates exponential from power-law behavior for the roughness.

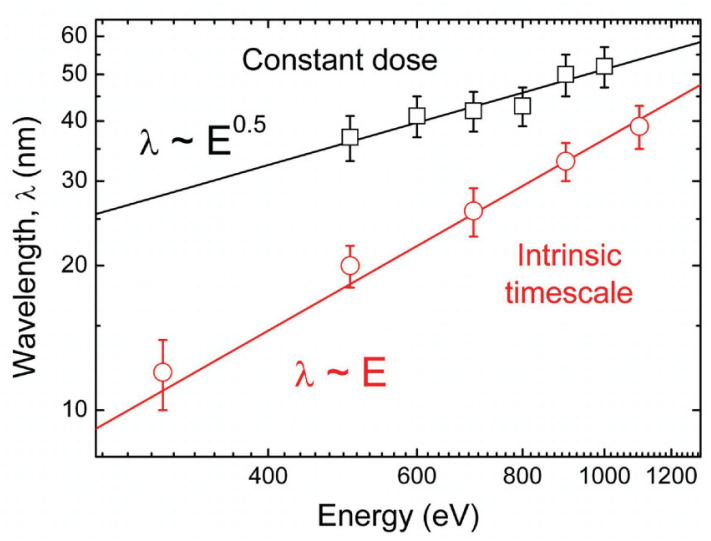

FIG. 6. (Color online) Ripple wavelength dependence on the ion energy for constant-dose experiments (squares) and intrinsic-timescale experiments (circles) for an incidence angle of $65^{\circ}$ with $J_{0}=$ 300 and $30 \mu \mathrm{A} \mathrm{cm}^{-2}$, respectively. The upper line is a power-law fit with exponent 0.5 , while the lower line is a linear fit validating the theoretical prediction given by Eq. (4).

Moreover, in Fig. 6 we show the dependence of the wavelength on the ion energy. Again, the prediction of the theory that $\lambda$ scales almost linearly with ion energy fits nicely, provided the experiments are performed at the intrinsic time scale, and not as (the customary) constant-dose experiments. In general, agreement is not reached for the latter since those data points correspond to the nonlinear regime that sets in for times longer than the scale (5), at which predictions made from the linear approximation break down. This fact proves the self-consistency of our present analysis and underscores the predictive power of the stress-driven solid flow theory. However, BH theory could still be relevant to explain the appearance of parallel ripples, although their appearance at large angles is still a matter of debate experimentally (see Ref. 24 for a recent discussion).

\section{DISCUSSION AND CONCLUSIONS}

We have presented theoretical predictions based on ioninduced residual stress and viscous flow as the main physical mechanisms driving surface nanopattern formation by IBS of silicon, together with the experimental validation of such predictions. These results allow us to extract important conclusions. Specifically, the predictions in terms of ion energy originate from the physical model in Ref. 30, in which direct knock-on implantation is assumed to produce stress that can be relaxed by defect migration to the free surface of the amorphous layer. Once this fact is taken as an input of our hydrodynamic framework, it allows us to provide the scaling of different observables with energy. Most remarkably, the ripple wavelength is predicted to scale roughly linearly with $E$, in marked contrast with the classical $E^{-1 / 2}$ scaling from $\mathrm{BH}$ theory. ${ }^{3,4,6}$ In retrospect, experimental scalings of the form $\lambda(E) \sim E^{p}$ with positive values of $p$ had been already reported previously, representative examples being, e.g., Refs. 22 and 23, which correspond to amorphizable targets. At a time in which the nontrivial patterning effects of impurities had not been universally acknowledged, and being incompatible with the $\mathrm{BH}$ paradigm, these cases were considered anomalous in 
some sense. ${ }^{3}$ Nonetheless, they prompted the formulation of non-BH mechanisms that were able to account for them in principle, such as, e.g., the so-called ion-induced effective smoothing, which already required invoking transport effects induced by the ion beam. ${ }^{40,41}$ These were later refined through more natural formulations as in Ref. 34; see an overview in Ref. 18. Nowadays, the BH mechanism has been unambiguously invalidated as the main operating mechanism for the class of targets studied in the present work, at least at nongrazing angles of incidence. In such a context, the assessment of a similar $\lambda \sim E$ law for a variety of materials including silica, ${ }^{22} \mathrm{HOPG}^{23}$ amorphous carbon, ${ }^{24}$ and (in our present case) silicon suggests that such a relation may be more the rule than the exception for amorphizable monoelemental targets, underscoring the generality of the mechanism of stress-induced viscous flow that has been validated here for the last system. Naturally, further enquiries are needed in which, e.g., other target-ion combinations and/or energy ranges are probed.
Finally, an important part of our analysis relates to the dependence of the linear or nonlinear behavior on experimental parameters and observation time. Thus, an essential requirement for experimental reproducibility and meaningful comparison with theory is that measurements made for different conditions (e.g., energy, angle, etc.) correspond to the proper dynamical regime. We expect that clarification of the physical basis of IBS will allow enhancement of experimental control over the technique, which will finally bring it up to the well-founded high expectations expressed almost 40 years ago. ${ }^{1}$

\section{ACKNOWLEDGMENTS}

This work has been partially supported by Grants No. FIS2009-12964-C05-01, No. FIS2009-12964-C05-03, No. FIS2009-12964-C05-04, and No. CSD2008-00023 (MICINN, Spain). J.M.-G. was supported by the Spanish MEC through the Juan de la Cierva program.
*Corresponding author: marioc@upcomillas.es

${ }^{1}$ N. Taniguchi, in Proceedings of the International Conference on Production Engineering (ICPE) (Japan Society of Precision Engineering, Tokyo, 1974).

${ }^{2}$ S. Facsko, T. Dekorsy, C. Koerdt, C. Trappe, H. Kurz, A. Vogt, and H. L. Hartnagel, Science 285, 1551 (1999).

${ }^{3}$ W. L. Chan and E. Chason, J. Appl. Phys. 101, 121301 (2007).

${ }^{4}$ J. Muñoz-García, L. Vázquez, R. Cuerno, J. A. Sánchez-García, M. Castro, and R. Gago, in Toward Functional Nanomaterials, edited by Z. Wang (Springer, New York, 2009).

${ }^{5}$ V. K. Smirnov, D. S. Kibalov, O. M. Orlov, and V. V. Graboshnikov, Nanotechnology 14, 709 (2003).

${ }^{6}$ R. Bradley and J. Harper, J. Vac. Sci. Technol. A 6, 2390 (1988).

${ }^{7}$ M. Cross and H. Greenside, Pattern Formation and Dynamics in Nonequilibrium Systems (Cambridge University Press, Cambridge, England, 2009).

${ }^{8}$ G. Ozaydin, A. S. Özcan, Y. Wang, F. Ludwig, H. Zhou, R. L. Headrick, and D. P. Siddons, Appl. Phys. Lett. 87, 163104 (2005).

${ }^{9}$ R. M. Bradley and P. D. Shipman, Phys. Rev. Lett. 105, 145501 (2010).

${ }^{10}$ C. S. Madi, B. Davidovitch, H. B. George, S. A. Norris, M. P. Brenner, and M. J. Aziz, Phys. Rev. Lett. 101, 246102 (2008).

${ }^{11}$ S. Macko, F. Frost, B. Ziberi, D. F. Förster, and T. Michely, Nanotechnology 21, 085301 (2010).

${ }^{12}$ G. Carter and V. Vishnyakov, Phys. Rev. B 54, 17647 (1996).

${ }^{13}$ M. Moseler, P. Gumbsch, C. Casiraghi, A. C. Ferrari, and J. Robertson, Science 309, 1545 (2005).

${ }^{14}$ C. S. Madi, E. Anzenberg, K. F. Ludwig, Jr., and M. J. Aziz, Phys. Rev. Lett. 106, 066101 (2011).

${ }^{15}$ S. A. Norris, J. Samela, L. Bukonte, M. Backman, F. Djurabekova, K. Nordlund, C. S. Madi, M. P. Brenner, and M. J. Aziz, Nat. Commun. 2, 276 (2011).
${ }^{16}$ M. Z. Hossain, K. Das, J. B. Freund, and H. T. Johnson, Appl. Phys. Lett. 99, 151913 (2011).

${ }^{17}$ M. Castro and R. Cuerno, arXiv:1007.2144.

${ }^{18}$ R. Cuerno, M. Castro, J. Muñoz-García, R. Gago, and L. Vázquez, Nucl. Instrum. Methods Phys. Res. Sect. B 269, 894 (2011).

${ }^{19}$ M. Castro and R. Cuerno, Appl. Surf. Sci. 258, 4171 (2012).

${ }^{20}$ S. A. Norris, Phys. Rev. B 85, 155325 (2012).

${ }^{21}$ S. A. Norris, Phys. Rev. B 86, 235405 (2012).

${ }^{22}$ C. C. Umbach, R. L. Headrick, and K.-C. Chang, Phys. Rev. Lett. 87, 246104 (2001).

${ }^{23}$ S. Habenicht, Phys. Rev. B 63, 125419 (2001).

${ }^{24}$ O. Bobes, K. Zhang, and H. Hofsäss Phys. Rev. B 86, 235414 (2012).

${ }^{25}$ H. Gnaser, Low Energy Ion Irradiation of Solid Surfaces (SpringerVerlag, New York, 1999).

${ }^{26}$ N. Kalyanasundaram, M. C. Moore, J. B. Freund, and H. T. Johnson, Acta Mater. 54, 483 (2006)

${ }^{27}$ N. Medhekar, W. Chan, V. Shenoy, and E. Chason, J. Phys.: Condens. Matter 21, 224021 (2009).

${ }^{28}$ E. Chason and V. Shenoy, Nucl. Instrum. Methods . Phys. Res. Sec. B 272, 178 (2012).

${ }^{29}$ H. Windischmann, J. Appl. Phys. 62, 1800 (1987).

${ }^{30}$ C. A. Davis, Thin Solid Films 226, 30 (1993).

${ }^{31}$ H. Hofsäss, H. Feldermann, R. Merk, M. Sebastian, and C. Ronning, Appl. Phys. A: Lasers Opt. 66, 153 (1998).

${ }^{32}$ B. Abendroth, H. Jäger, W. Möller, and M. Bilek, Appl. Phys. Lett. 90, 181910 (2007).

${ }^{33}$ B. Ziberi, F. Frost, T. Höche, and B. Rauschenbach, Phys. Rev. B 72, 235310 (2005).

${ }^{34}$ M. Castro, R. Cuerno, L. Vázquez, and R. Gago, Phys. Rev. Lett. 94, 016102 (2005).

${ }^{35}$ J. Muñoz-García, M. Castro, and R. Cuerno, Phys. Rev. Lett. 96, 086101 (2006) 
${ }^{36}$ J. Muñoz-García, R. Cuerno, and M. Castro, Phys. Rev. B 78, 205408 (2008).

${ }^{37}$ A. Keller, S. Roßbach, S. Facsko, and W. Möller, Nanotechnology 19, 135303 (2008).

${ }^{38}$ L. Sedov, Similarity and Dimensional Methods in Mechanics (CRC Press, Boca Raton, FL, 1993).
${ }^{39}$ The fit can be further improved (not shown) if one also fits the critical angle as $\lambda=2 \pi \sqrt{\left[2 \sigma / f_{E} \phi^{\prime}\left(\theta_{c}\right)\right]}\left(\theta-\theta_{c}\right)^{-1 / 2}$.

${ }^{40}$ M. Makeev and A.-L. Barabási, Appl. Phys. Lett. 71, 2800 (1997).

${ }^{41}$ M. Makeev, R. Cuerno, and A.-L. Barabási, Nucl. Instrum. Methods Phys. Res. Sect. B 197, 185 (2002). 